\title{
Diseño y simulación de un controlador difuso de temperatura empleando el concresor basado en relaciones booleanas
}

\section{Design and simulation of a fuzzy temperature controller using the concresor based on boolean relationship}

\section{Helbert Eduardo Espitia C.}

Ingeniero Electrónico, ingeniero Mecatrónico, especialista en Telecomunicaciones Móviles, magíster en Ingeniería Industrial, magíster en Ingeniería Mecánica. Docente de la Universidad Distrital Francisco José de Caldas. Bogotá, Colombia. heespitiac@udistrital.edu.co

\section{José JaIro Soriano MéndeZ} Ingeniero Químico, ingeniero Electrónico, magíster en Ingeniería Industrial. Docente de la Universidad Distrital Francisco José de Caldas. Bogotá, Colombia. jairosoriano@udistrital.edu.co

Fecha de recepción: Agosto 30 de 2011

Clasificación del artículo: Investigación (Conciencias) Fecha de aceptación: Noviembre 28 de 2011

Palabras clave: $\mathrm{CBR}$, control, lógica Booleana, lógica difusa, sistema térmico.

Key words: CBR, control, Boolean logic, fuzzy logic, thermal system.

\section{RESUMEN}

Este trabajo propone el análisis y diseño de un controlador difuso de temperatura, usando el concresor, basado en relaciones Booleanas (CBR). En la primera parte, se presenta el diseño del controlador Booleano. Posteriormente, se efectúa el diseño del controlador difuso partiendo del con- trolador Booleano realizado anteriormente. Las superficies de control obtenidas con y sin simplificación de términos evidencian las características que tiene la metodología propuesta. Adicionalmente, se analizan los efectos que tienen las simplificaciones realizadas en las reglas de control. 


\section{con-ciencias}

\section{ABSTRACT}

This paper proposes the analysis and design of a Temperature Fuzzy controller using concresor based on Boolean relations (CBR). Firstly, presents the design of a Boolean controller. Later, fuzzy controller is designed based on Boolean controller raised previously. The control surfaces with and without simplification, show the features of proposes methodology. Additionally we analyze the effects of the simplifications made in the control rules.

\section{INTRODUCCIÓN}

La lógica difusa presenta gran aplicabilidad en los sistemas de control, debido a su flexibilidad para la implementación de estrategias de control [1], [2]. Por otro lado la lógica Booleana es una herramienta útil en el diseño de sistemas automáticos [3], [4]. Sin embargo, los sistemas de control basados en lógica Booleana presentan un desempeño limitado debido a transiciones bruscas de las diferentes acciones de control. Una forma de mejorar el desempeño de estos sistemas consiste en reemplazar los conjuntos Booleanos por difusos. Un trabajo donde se busca aprovechar las características del diseño de automatismos basados en álgebra Booleana se presenta en [5], donde se propone un método de minimización de expresiones en sistemas de inferencia difusa, empleando lógica Booleana. Otra orientación con un sentido más práctico se presentó en [6] y [7], donde se plantea una metodología basada en lógica Booleana empleando conjuntos difusos para lograr la implementación del proceso asociado con la defusificación, posteriormente en [8] se considera que esta propuesta se podía interpretar como un sistema de inferencia difusa por sí mismo.

El Concresor Basado en Relaciones Booleanas (CBR) o como se denominó originalmente en inglés Defuzification Based on Boolean Relations (DBR), busca plantear un mecanismo de implementación para los sistemas de lógica difusa, tomando como referencia el diseño de automatismos, ya que estos sistemas son muy empleados en control de procesos por su facilidad de implementación. Esta técnica de diseño considera los sensores, actuadores y las relaciones Booleanas empleadas en las estrategias de control [7].

Por otro lado, uno de los problemas prácticos y comunes en ingeniería es el control de temperatura. Un enfoque que se ha realizado para la solución de este tipo de problemas consiste en la implementación de controladores basados en lógica difusa, donde algunos ejemplos de estas soluciones se encuentran en [9], [10], [11] y [12]. En este documento se considera el control de un sistema de temperatura similar al presentado en [10], [11] y [13].

La metodología de diseño empleada, consiste en realizar una aplicación desde un punto de vista Booleano y luego emplear conjuntos difusos para tener una implementación tipo CBR. Sobre los aspectos a considerar en el diseño, se tiene la posibilidad de emplear los casos no importa para la implementación del controlador.

\section{CONCRESOR BASADO EN RELACIONES BOOLEANAS}

El concresor basado en relaciones Booleanas (CBR) busca emplear el proceso de diseño que tienen los sistemas basados en álgebra Booleana para la implementación de controladores difusos. Como primer aspecto a considerar se tiene la relación (isomorfismo) [14], observada entre teoría de conjuntos, lógica y sistemas matemáti- 
Tabla 1. Equivalencia entre conjuntos, lógica y algebra Booleana.

\begin{tabular}{|c|c|c|}
\hline Conjuntos & Lógica & $\begin{array}{c}\text { Algebra } \\
\text { Booleana }\end{array}$ \\
\hline Pertenencia & Veracidad & Valor \\
Pertenece $(\epsilon)$ & Verdad $(V)$ & 1 \\
No pertenece $(\notin)$ & Falso $(F)$ & 0 \\
Intersección $(\neg)$ & Conjunción $(\wedge)$ & Producto $(\times)$ \\
Unión $(\cup)$ & Disyunción $(\vee)$ & Suma $(+)$ \\
Complemento $(-)$ & Negación $(\neg)$ & Complemento (') \\
\hline
\end{tabular}

cos (retículos y álgebra Booleana) [15], algunas de las equivalencias más importantes de estos isomorfismos se pueden observar en la Tabla 1.

La implementación de sistemas de inferencia basados en relaciones Booleanas parte del esquema para la formulación de controladores de tipo Booleano. Desde el punto de vista de lógica, el controlador basado en lógica Booleana, se puede considerar como un sistema de inferencia, con reglas de la forma:

$$
\operatorname{Si},\left(\hat{A}_{1} \wedge \hat{A}_{2} \wedge \ldots \wedge \hat{A}_{p}\right), \text { Entonces, } Y_{k}
$$

Donde,

$$
\hat{A}_{p}=\left\{\begin{array}{l}
A_{p}, s i, \hat{A}_{p}=1, \\
\bar{A}_{p}, s i, \hat{A}_{p}=0 .
\end{array}\right.
$$

Con la finalidad de mejorar el desempeño del controlador Booleano se cambian los conjuntos Booleanos por difusos, de tal forma que se puedan tener transiciones suaves y monótonas entre regiones Booleanas. Debido a lo anterior es necesario definir las operaciones de intersección y unión para conjuntos difusos, las cuales son respectivamente: t-normas ( ) y s-normas ( ) [5], los anteriores operadores deben cumplir las siguientes propiedades:

$$
\begin{aligned}
& a \otimes(b \otimes c)=(a \otimes b) \otimes c \\
& a \oplus(b \oplus c)=(a \oplus b) \oplus c \\
& a \otimes b=b \otimes a \\
& a \oplus b=b \oplus a \\
& a \otimes 1=a \\
& a \oplus 0=a
\end{aligned}
$$

En el proceso de síntesis de controladores Booleanos se suele emplear la propiedad de la Ec. (4).

$$
(a \cap \bar{b}) \cup(a \cap b)=a \cap(b \cup \bar{b})=a
$$

Para extender este principio a conjuntos difusos se debe asumir la Ec. (5), [5].

$$
(a \otimes b) \oplus(a \otimes c)=a \otimes(b \oplus c)
$$

Es importante tener presente que para conjuntos difusos, se tienen las propiedades mostradas en la Ec. (6).

$$
\begin{aligned}
& a \oplus \bar{a} \leq 1 \\
& a \otimes \bar{a} \geq 0
\end{aligned}
$$

\subsection{Sistemas de inferencia difusa basados en relaciones Booleanas}

Para un sistema de $w$ funciones de activación, $p$ conjuntos, $q$ posibles implicaciones, siendo $Y_{m}$ la $m$-ésima salida de activación y $\mu_{\hat{A}_{j}^{k}}$ la $j$ -ésima función de pertenencia de la $k$-ésima implicación, el proceso de inferencia de $Y_{m}$, considerando conjuntos booleanos o difusos, se puede expresar como se muestra en la Ec. (7):

$$
Y_{m}=\bigcup_{k \mid f_{k, m}}^{q} \bigcap_{j=1}^{p} \hat{A}_{j}^{k}
$$

En el caso de considerar funciones de pertenencia de conjuntos difusos se tiene la siguiente expresión:

$$
Y_{m}=\bigoplus_{k \mid y_{k, m}=1}^{q} \bigotimes_{j=1}^{p} \mu_{\hat{A}_{j}^{k}}
$$


Tanto para conjuntos Booleanos como difusos la salida concreta del sistema se puede calcular como:

$$
y=\sum_{m=1}^{w} Y_{m} v_{m}
$$

donde $v_{m}$ corresponde al $m$-ésimo actuador virtual, entonces la $m$-ésima salida virtual es:

$$
y_{v_{m}}=Y_{m} v_{m}
$$

\subsection{Salida virtual}

En el diseño de automatismos, la salida se puede considerar como el consecuente de una función Booleana de las entradas que corresponden a los antecedentes. El sistema de inferencia difusa basado en relaciones Booleanas toma este esquema, el cual es el mismo en lógica difusa, sin embargo, la salida desde el punto de vista de automatismos se considera como la acción total que se tiene por parte de los actuadores que están afectando el sistema. En el caso donde solo se dispone de un elemento de acción, este se puede considerar como la suma de varios actuadores los cuales se denominan virtuales y que a su vez tienen asociada una salida virtual.

Dependiendo de los conjuntos empleados y de las acciones a realizar en las regiones de operación, es posible tener un solapamiento de las acciones, por lo cual, la salida total puede ser mayor que las acciones virtuales parciales.

En esta propuesta los actuadores virtuales son constantes y en un esquema tradicional de lógica difusa tipo Mamdani se pueden considerar como conjuntos singleton (univaluados) en el universo de discurso de salida. Dependiendo el caso, los actuadores virtuales pueden ser positivos o negativos y sus valores también pueden ser definidos según la aplicación.

Para el sistema de inferencia, la salida corresponde a la suma de las respectivas salidas virtuales:

$$
y=y_{v_{1}}+y_{v_{2}}+\ldots+y_{v_{m}}+\ldots+y_{v_{w}}=\sum_{m=1}^{w} y_{v_{m}}
$$

donde $y_{v_{m}}$ esta dado por la Ec. (10). El término de salida virtual fue concebido bajo una correspondencia física real en aplicaciones de control, para la salida total del sistema de inferencia. A manera de ilustración del concepto de salida virtual, se puede observar la acción que tiene una válvula de para el llenado de un tanque; en este caso el flujo permitido por la válvula se puede considerar como el aporte que darían tres válvulas virtuales (que tienen una existencia aparente y no real) de diferente diámetro que dan lugar a los actuadores virtuales:

- Flujo pequeño: $v_{1}=05^{\prime \prime}$

- Flujo mediano: $v_{2}=10^{\prime \prime}$

- Flujo grande: $v_{3}=15^{\prime \prime}$

\section{SIMPLIFICACIÓN DE REGLAS DE INFERENCIA}

La síntesis de automatismos aplicando álgebra de Boole permite tener implementación de sistemas más simples, al respecto, se han realizado estudios sobre esta característica para la optimización de sistemas de inferencia difusa [5]. Por otro lado, en [16] se propone un método para la simplificación de reglas de inferencia, en sistemas de lógica difusa tipo Mamdani.

A continuación se analizan las características importantes de la síntesis Booleana aplicada a conjuntos difusos, las restricciones matemáticas y los criterios de implementación prácticos de los sistemas de lógica difusa basados en el CBR siendo una de las características interesantes la capacidad de realizar simplificación de sentencias mediante herramientas de lógica Booleana, como son los mapas de Karnaugh.

Con la finalidad de tener una simplificación de términos se puede plantear un conjunto de restric- 
ciones con las cuales las funciones de pertenencia cumplan las condiciones de simplificación. Lo anterior surge considerando el concepto de conjunto Booleano bajo la perspectiva de retículo Booleano [15], [17].

La propuesta de la simplificación Booleana aplicada a conjuntos difusos se encuentra basada en el concepto de conjunto ordenado definido como retículo (lattice) [15], [17]. Un retículo es comúnmente expresado como $\langle L ; \vee, \lambda$, siendo $\vee$ el operador de unión (supremo) y $\wedge$ el operador de intersección (ínfimo). Lo anterior resulta ser adecuado ya que el álgebra de Boole también presenta una definición como retículo Booleano [15].

Una de las características que presentan los retículos en relación con el orden de sus elementos establece que si $a \leq b$ entonces $a \vee b=b$ y $a \wedge b=a$. Siendo de interés la segunda propiedad, ya que esta fundamenta la ley del tercer excluido en álgebra Booleana.

Para encontrar los requerimientos necesarios que permitan aplicar la simplificación de la metodología Booleana con conjuntos difusos, es necesario examinar la propiedad del álgebra Booleana asociada a esta característica. Del álgebra Booleana se observa que la propiedad que permite la reducción de términos se fundamenta en la ley del tercer excluido,

$$
\begin{aligned}
& B \cup \bar{B}=1 \\
& B \cap \bar{B}=0
\end{aligned}
$$

la cual se emplea para obtener una simplificación de términos gracias a:

$$
A \cap(B \cup \bar{B})=A
$$

aunque la reducción de términos se logra empleando álgebra Booleana y conjuntos Booleanos, es posible considerar una situación similar cuando se están empleando conjuntos difusos. Aunque no sea posible contemplar la reducción Booleana en forma general para conjuntos difusos por no cumplir con la ley del tercer excluido:

$$
\begin{aligned}
& \mu_{B} \oplus \mu_{\bar{B}} \leq 1 \\
& \mu_{B} \otimes \mu_{\bar{B}} \geq 0
\end{aligned}
$$

se puede plantear el caso para el cual la reducción sea válida, es decir:

$$
\mu_{A} \oplus\left(\mu_{B} \oplus \mu_{\bar{B}}\right)=\mu_{A}
$$

lo cual es necesario para la simplificación de términos en álgebra Booleana, por tanto, al tener conjuntos difusos que cumplan con este requerimiento es posible realizar la simplificación de términos bajo la metodología Booleana empleando conjuntos difusos. Es importante señalar que una t-norma y s-norma que permiten el cumplimiento de la anterior relación son el mínimo y el máximo respectivamente [2].

Según el anterior planteamiento, para que la simplificación se pueda realizar se debe cumplir con:

$$
\mu_{A} \leq\left(\mu_{B} \oplus \mu_{\bar{B}}\right)
$$

Adicionalmente, con la finalidad de emplear la metodología de síntesis Booleana es necesaria la propiedad distributiva [5]:

$$
\mu_{A} \otimes\left(\mu_{B} \oplus \mu_{C}\right)=\left(\mu_{A} \otimes \mu_{B}\right) \oplus\left(\mu_{A} \otimes \mu_{C}\right)
$$

\subsection{Simplificación de variables}

Considerando $n$ variables $A_{1}, A_{2}, \ldots, A_{n}$ a simplificar y una función $Y_{B}$ correspondiente a la intersección Booleana de $m$ variables $Y_{B}=B_{1} \cap B_{2} \cap, \cdots, \cap B_{m}$, entonces, es posible tener un caso donde:

$$
Y=Y_{B} \cap\left[\left(A_{1} \cup \bar{A}_{1}\right) \cap\left(A_{2} \cup \bar{A}_{2}\right) \cap \cdots \cap\left(A_{n} \cup \bar{A}_{n}\right)\right]
$$


En este caso, la intersección de los términos formados por la unión de una variable y su complemento generan la unión de $2^{n}$ intersecciones producidas al aplicar la propiedad distributiva de la intersección Booleana sobre la unión Booleana, siendo las variables simplificadas las presentes en el anterior proceso. La respectiva restricción que se genera para esta simplificación es:

$$
Y_{B} \subseteq\left[\left(A_{1} \cup \bar{A}_{1}\right) \cap\left(A_{2} \cup \bar{A}_{2}\right) \cap \cdots \cap\left(A_{n} \cup \bar{A}_{n}\right)\right]
$$

A medida de ejemplo se tiene la simplificación general de una variable $A$ donde se considera que existe una salida parcial $Y_{B}$ correspondiente a la intersección Booleana de posibles variables $Y_{B}=B_{1} \cap B_{2} \cap \cdots \cap B_{n}$, entonces, se tiene un caso donde la función de salida es

$$
\begin{gathered}
Y=\left(Y_{B} \cap \bar{A}\right) \cup\left(Y_{B} \cap A\right) \\
Y=Y_{B} \cap(\bar{A} \cup A)
\end{gathered}
$$

por lo tanto, la condición para que se cumpla la simplificación de la variable $A$ es:

$$
Y_{B} \subseteq(\bar{A} \cup A)
$$

en el caso de emplear conjuntos difusos se tiene:

$$
\mu_{Y_{B}} \leq \max \left\{\left(1-\mu_{A}\right), \mu_{A}\right\}
$$

\section{METODOLOGÍA}

El sistema consiste en un control de temperatura para una bañera, este es un problema que se ha trabajado en [11]. Las especificaciones del sistema son: capacidad del tanque $15 \mathrm{~L}\left(0,015 \mathrm{~m}^{3}\right)$, temperatura ambiente del agua $25^{\circ} \mathrm{C}$, potencia de calefacción $2.500 \mathrm{~W}$, flujo del agua $1 \mathrm{~L} / \mathrm{min}$ $\left(1,6 \times 10^{-5} \mathrm{~m}^{3} / \mathrm{s}\right)$. La Fig. 1 muestra el esquema del sistema térmico considerado.

Las variables y parámetros del sistemas son:

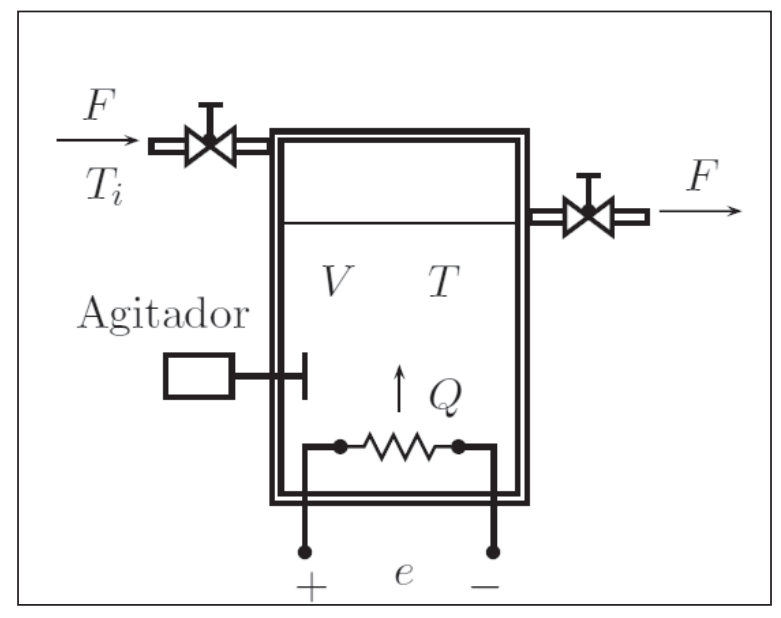

Fig. 1: Esquema del sistema térmico.

- $\dot{m}$ : Flujo másico.

- $T$ : Temperatura del tanque.

- $F$ : Flujo del agua.

- $T_{i}$ : Temperatura ambiente del agua.

- $V$ : Volumen del tanque.

- $Q$ : Calor de entrada.

- $C_{p}$ : Calor especifico.

- $\rho$ : Densidad.

El calor específico del agua es $1 \mathrm{cal} /\left({ }^{\circ} \mathrm{C} \cdot \mathrm{g}\right)=$ $4.186,8 \mathrm{~J} /\left({ }^{\circ} \mathrm{C} \cdot \mathrm{Kg}\right)$ y la respectiva densidad es, de $1.000 \mathrm{Kg} / \mathrm{m}^{3}$.

El balance de energía del sistema es:

$$
\dot{m} C_{p} T_{i}+Q=\rho V C_{p} \frac{d T}{d t}+\dot{m} C_{p} T
$$

por lo tanto el proceso de calentamiento está dado por la siguiente expresión:

$$
\frac{d T}{d t}=\frac{F\left(T_{i}-T\right)}{V}+\frac{Q}{\rho V C_{p}}
$$




\subsection{Diseño Booleano}

Una primera alternativa de control como la existente en muchos equipos de calefacción comerciales, consiste en un automatismo que emplea sensores de tipo bivalente, es decir, los valores de pertenencia que toma cada una de las variables de medición son 1 o 0 .

Los conjuntos involucrados en los universos de discurso que se consideran son: integral de error positivo $\mu_{p}(i e)$, integral de error negativo $\mu_{n}(i e)$, error positivo $\mu_{p}(e)$ y error negativo $\mu_{n}(e)$. En la Fig. 2 se presentan los conjuntos Booleanos.

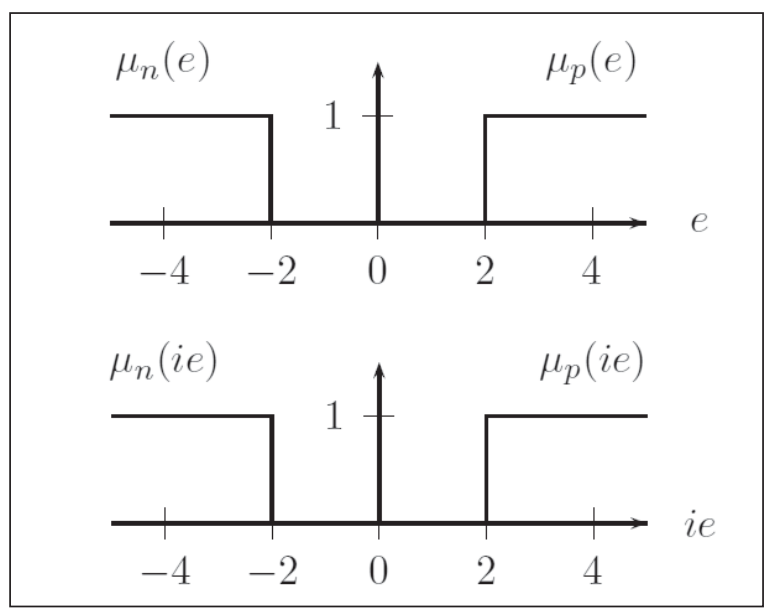

Fig. 2. Conjuntos Booleanos para el control de temperatura.
Donde:

$$
\begin{gathered}
e=T_{r}-T \\
i e=\int_{0}^{t} e(\tau) d \tau
\end{gathered}
$$

en el caso discreto se tiene:

$$
i e_{t}=i e_{t-1}+e \Delta t
$$

La base de reglas se propone según la información obtenida, del comportamiento del sistema.

Los actuadores virtuales considerados en este caso son:

- $v_{P g}$ : Energía positiva grande.

- $v_{P p}$ : Energía positiva pequeña.

Con los anteriores actuadores virtuales, los valores de energía que se esperan tener en la salida del sistema son:

- Energía alta: $\quad y=v_{P g}+v_{P p}$

- Energía pequeña: $\quad y=v_{P p}$

Para lograr los diferentes niveles de energía en la salida, se emplean las funciones de activación Booleanas $Y_{P g}$ y $Y_{P p}$, de tal forma que la salida se puede calcular como:

$$
y=Y_{P g} v_{P g}+Y_{P p} v_{P g}
$$

Tabla 2. Reglas lingüísticas para el control de temperatura.

\begin{tabular}{|l|l|l|l|l|}
\hline Si Error positivo & e & integral de error positivo & entonces & energía alta \\
\hline Si Error positivo & e & integral de error negativo & entonces & energía pequeña \\
\hline Si Error negativo & e & integral de error positivo & entonces & energía nula \\
\hline Si Error negativo & e & integral de error negativo & entonces & energía nula \\
\hline Si Error cero & e & integral de error cero & entonces & energía nula \\
\hline Si Error cero & e & integral de error negativo & entonces & energía nula \\
\hline Si Error cero & e & integral de error positivo & entonces & energía nula \\
\hline Si Error negativo & e & integral de error cero & entonces & energía nula \\
\hline Si Error positivo & e & integral de error cero & entonces & energía nula \\
\hline
\end{tabular}


Los valores de los actuadores virtuales son; $v_{P g}=500 \mathrm{~W}$ y $v_{P g}=1500 \mathrm{~W}$.

La tabla de reglas lingüísticas (Tabla 2) también se puede observar como un cuadro de relaciones para las regiones del universo de discurso del error y la integral del error, Tabla 3. En el cuadro de relaciones se observa que no existe acción de control negativa para el sistema, por lo cual esta acción queda sujeta a la inercia propia del sistema. Otro aspecto de importancia consiste en la falta de una acción en la zona de cero, siendo esto relevante al momento de plantear los conjuntos difusos.

Tabla 3. Cuadro de relaciones para el error y la integral del error.

\begin{tabular}{|l|c|c|c|}
\hline \multicolumn{1}{|c|}{ ie $\backslash$ e } & Negativo & Cero & Positivo \\
\hline Negativo & Nulo & Nulo & $Y_{P p}$ \\
\hline Cero & Nulo & Nulo & Nulo \\
\hline Positivo & Nulo & Nulo & $Y_{P g}, Y_{P p}$ \\
\hline
\end{tabular}

Con la finalidad de tener una representación compacta, se propone la siguiente nomenclatura para los conjuntos Booleanos:

$$
\begin{aligned}
& -e_{p}=\mu_{p}(e) \\
& \text { - } e_{n}=\mu_{n}(e) \\
& \text { - } i e_{p}=\mu_{p}(i e) \\
& \text { - } i e_{n}=\mu_{n}(i e)
\end{aligned}
$$

Las reglas lingüísticas se pueden representar mediante una tabla de verdad con la finalidad de encontrar las respectivas relaciones Booleanas de las salidas de activación. La Tabla 4 representa las reglas Booleanas para el sistema de control. Los términos faltantes de la Tabla 4 se consideran como casos no importa.
Tabla 4. Tablade verdad para el control de temperatura.

\begin{tabular}{|c|c|c|c|c|c|}
\hline$e_{p}$ & $e_{n}$ & $i e_{p}$ & $i e_{n}$ & $Y_{P g}$ & $Y_{P_{p}}$ \\
\hline 0 & 1 & 0 & 1 & 0 & 0 \\
\hline 0 & 1 & 1 & 0 & 0 & 0 \\
\hline 1 & 0 & 0 & 1 & 0 & 1 \\
\hline 1 & 0 & 1 & 0 & 1 & 1 \\
\hline 0 & 0 & 0 & 0 & 0 & 0 \\
\hline 0 & 0 & 1 & 0 & 0 & 0 \\
\hline 0 & 0 & 0 & 1 & 0 & 0 \\
\hline 1 & 0 & 0 & 0 & 0 & 0 \\
\hline 0 & 1 & 0 & 0 & 0 & 0 \\
\hline
\end{tabular}

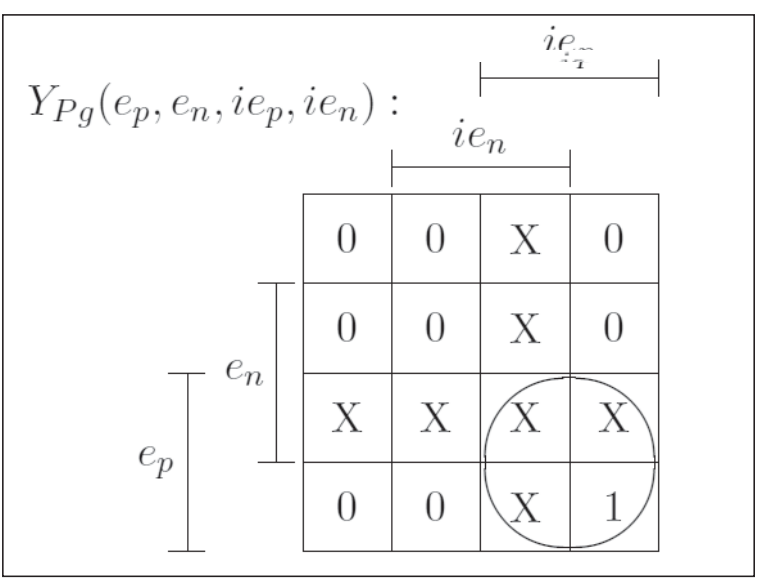

Fig. 3. Mapa de Karnaugh para la función de activación $Y_{P g}$.

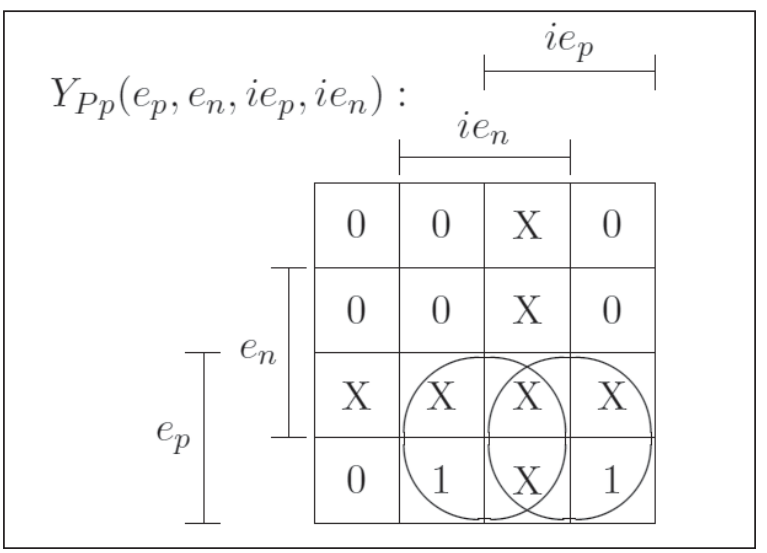

Fig. 4. Mapa de Karnaugh para la función de activación $Y_{P g}$. 
Empleando los mapas de Karnaugh de las Fig. 3 y 4, se pueden encontrar las ecuaciones simplificadas para $Y_{P g}$ y $Y_{P p}$.

$$
\begin{gathered}
Y_{P g}=e_{p} \cap i e_{p} \\
Y_{P p}=\left(e_{p} \cap i e_{p}\right) \cup\left(e_{p} \cap i e_{n}\right)
\end{gathered}
$$

En la Fig. 5 se presenta la superficie de control para el controlador Booleano simplificado y no simplificado. La superficie de control para el caso Booleano es la misma con y sin reglas simplificadas.

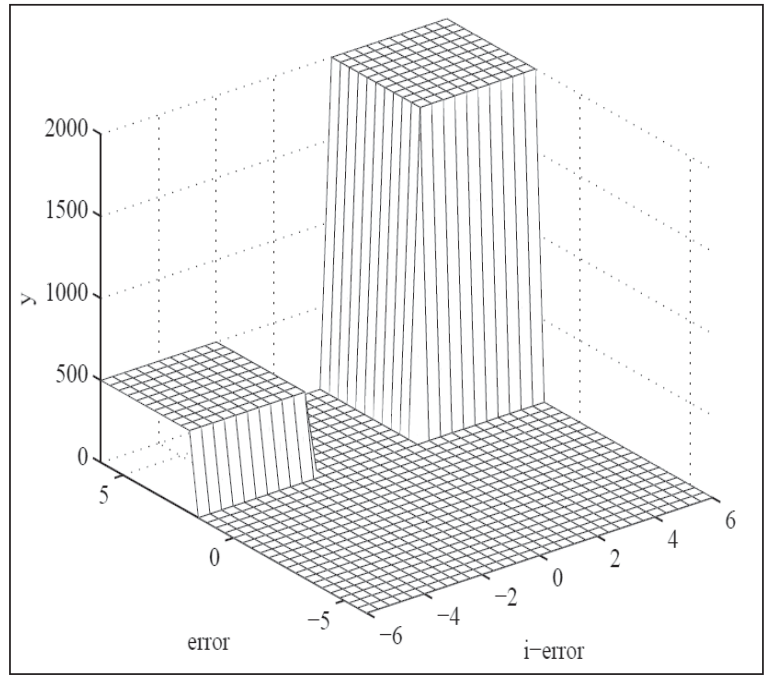

Fig. 5. Superficie de control para el controlador Booleano.

\subsection{Implementación CBR}

Luego de tener las reglas Booleanas asociadas a los conjuntos Booleanos, se procede a convertir estos conjuntos en difusos. La conversión se realiza considerando un nivel de $\alpha$-corte igual a 0,5 . El valor de $\alpha$-corte permite recuperar los conjuntos Booleanos al ser aplicado el mismo $\alpha$-corte en los anteriores conjuntos difusos.

Tomando funciones lineales para la construcción de los conjuntos difusos, se proponen los conjuntos de la Fig. 6.
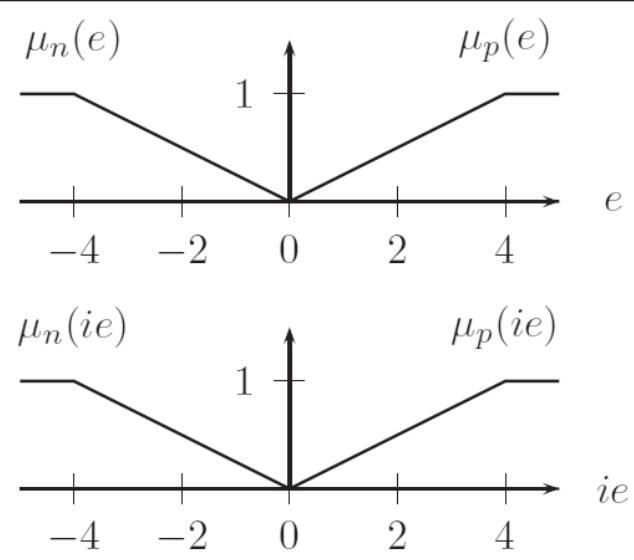

Fig. 6. Conjuntos difusos propuestos para el control de temperatura.

Para la implementación del CBR es necesario realizar una generalización de los operadores Booleanos a operadores difusos de s-normas y tnormas.

La implementación de las relaciones difusas se efectúa usando el operador máximo como s-norma, el operador mínimo como t-norma y el operador complemento como: $\mu_{\bar{A}}=1-\mu_{A}$.

$$
\begin{gathered}
Y_{P g}=\min \left[\mu_{p}(e), \mu_{p}(i e)\right] \\
Y_{P_{p}}=\max \left\{\min \left[\mu_{p}(e), \mu_{p}(i e)\right], \min \left[\mu_{p}(e), \mu_{n}(i e)\right]\right\}
\end{gathered}
$$

\subsection{Análisis de restricciones}

Considerando las ecuaciones a implementar, se presenta el siguiente grupo de restricciones.

Para la salida de activación $Y_{P g}$ se tiene:

$$
\begin{gathered}
Y_{P g}=\mu_{p}(e) \otimes \mu_{p}(i e) \\
\mu_{p}(e) \otimes \mu_{p}(i e) \leq\left(\mu_{n}(e) \oplus \mu_{\bar{n}}(e)\right) \otimes\left(\mu_{n}(i e) \oplus \mu_{\bar{n}}(i e)\right)
\end{gathered}
$$

la anterior restricción por estar en dos universos de discurso se puede separar en 


$$
\begin{gathered}
\mu_{p}(e) \leq \mu_{n}(e) \oplus \mu_{\bar{n}}(e) \\
\mu_{p}(i e) \leq \mu_{n}(i e) \oplus \mu_{\bar{n}}(i e)
\end{gathered}
$$

Para la otra salida de activación $Y_{P p}$ se tiene:

$$
Y_{P p}=\left(\mu_{p}(e) \otimes \mu_{p}(i e)\right) \oplus\left(\mu_{p}(e) \otimes \mu_{n}(i e)\right)
$$

como la restricción de $\mu_{p}(e) \otimes \mu_{p}(i e)$ ya fue considerada, entonces, se analiza el otro término $\mu_{p}(e) \oplus \mu_{n}(i e)$,

$$
\mu_{p}(e) \oplus \mu_{n}(i e) \leq\left(\mu_{n}(e) \oplus \mu_{\bar{n}}(e)\right) \otimes\left(\mu_{p}(i e) \oplus \mu_{\bar{p}}(i e)\right)
$$

la anterior restricción se pueden separa en

$$
\begin{gathered}
\mu_{p}(e) \leq \mu_{n}(e) \oplus \mu_{-}(e) \\
\mu_{n}(i e) \leq \mu_{p}(i e) \oplus \mu_{p}^{-}(i e)
\end{gathered}
$$

Finalmente del anterior análisis las restricciones que se deben cumplir son:

$$
\begin{gathered}
\mu_{p}(e) \leq \mu_{n}(e) \oplus \mu_{n}^{-}(e) \\
\mu_{p}(i e) \leq \mu_{n}(i e) \oplus \mu_{n}(i e) \\
\mu_{n}(i e) \leq \mu_{p}(i e) \oplus \mu_{p}^{-}(i e)
\end{gathered}
$$

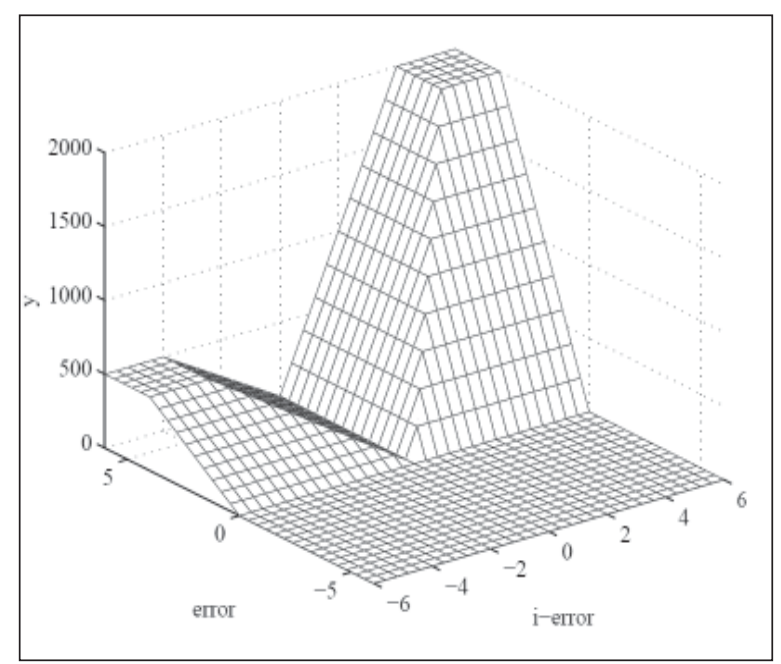

Fig. 7. Superficie de control para la implementación CBR con y sin simplificación de reglas.
Los conjuntos difusos propuestos en la Fig. 6 cumplen las anteriores restricciones, por lo tanto la superficie de control con y sin simplificación de reglas es la misma, tal como se observa en Fig. 7.

\section{RESULTADOS}

Para obtener la respuesta dinámica del sistema se implementó el controlador Booleano y CBR, tomando una referencia de $35^{\circ} \mathrm{C}$. La Fig. 7 muestra la respuesta del sistema para el controlador Booleano y CBR. En esta figura se observa que el controlador CBR presenta un mejor comportamiento que el controlador Booleano.

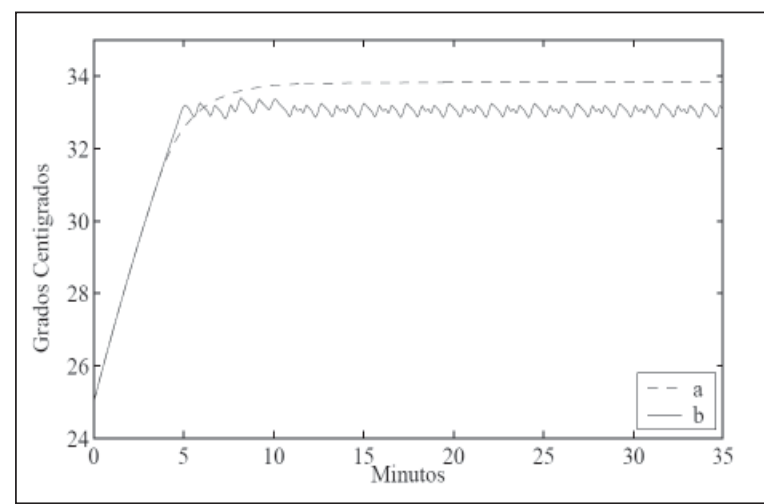

Fig. 8. Simulación del sistema para una referencia de $35^{\circ} \mathrm{C}$ : a) Controlador CBR, b) Controlador Booleano.

Considerando una referencia de $35^{\circ} \mathrm{C}$, los índices de desempeño del sistema se pueden apreciar en la Tabla 5.

Tabla 5. Indices de desempeño del sistema para una referencia de $35^{\circ} \mathrm{C}$ : a) Controlador $\mathrm{CBR}$, b) Controlador Booleano.

\begin{tabular}{|c|c|c|}
\hline Índice $\backslash$ Control & a & b \\
\hline$\% e_{s}$ & $3,96 \%$ & $6,50 \%$ \\
\hline$T_{s}$ & $11,34 \mathrm{mim}$ & $5,04 \mathrm{mim}$ \\
\hline
\end{tabular}




\section{con-ciencias}

\section{CONCLUSIONES}

Con la finalidad de mejorar el comportamiento dinámico del sistema se propone emplear conjuntos Booleanos adyacentes; empleando estos conjuntos se eliminan regiones que relacionan el error y la integral del error. Una mala identificacion de casos no importa, puede cambiar el cuadro de relaciones alterando el funcionamiento del sistema.

Dentro de la propuesta de controladores basados en concreción Booleana, es de gran importancia la forma de los conjuntos difusos de los universos de entrada. Existe una relación directa entre la forma de los conjuntos difusos y la respuesta final del controlador.

\section{REFERENCIAS}

[1] R. Babuska, Fuzzy Modeling for Control. Boston: Kluwer Academia Publishers, 1998.

[2] G. Klir and B. Yuan, Fuzzy Sets and Fuzzy Logic. New Jersey: Prentice Hall, 1995.

[3] E. Dougherty and C. Giardina, Mathematical Methods for Artificial Intelligence and Autonomous Systems. Prentice-Hall, 1988.

[4] K. Ogata, Dinámica de Sistemas. PrenticeHall Hisp, 1987.

[5] R. Rovatti, R. Guerrieri and G. Baccarani, "An Enhanced Two-Level Boolean Synthesis Methodology for Fuzzy Rules Minimization”, IEEE Transactions On Fuzzy Systems, vol. 3, no. 3, pp. 288 - 299, 1995.

[6] A. Ballén y C. Rodríguez, Diseño e implementación de un controlador difuso autosintonizado sobre microcontroladores, aplicado al control del péndulo invertido. Tesis de pregrado, Ingeniería Electrónica, Universidad Distrital Francisco José de Caldas, 2003.

[7] J. Soriano, O. González, F. Munar y A. Ramos, "Propuesta de concresor basado en relaciones Booleanas", Revista de Ingeniería, Universidad Distrital Francisco José de Caldas, vol. 6, no. 1, pp. 42-50, 2001.
[8] H. Espitia, Aplicación del concresor basado en relaciones Booleanas para sistemas de lógica difusa tipo dos, Tesis de Maestria Universidad Distrital Francisco José de Caldas, 2009.

[9] T. Acevedo, C. Martínez y J. Díaz, "Diseño de un Controlador Lógico Difuso Para Sistema de Calefaccion", Revista Colombiana de Tecnologías de Avanzada, vol. 2, no. 10, 10 pp. $53-58,2007$.

[10] Z. Gao and T. Trautzsch, "A Stable SelfTuning Fuzzy Logic Control System for Industrial Temperature Regulation", IEEE Transactions on Industry Applications, vol. 38, no. 2, pp. 414 - 424, 2002.

[11] G. Kumar, S. Chandra and B. Surekha, "A Genetic Based Neuro-Fuzzy Controller for Thermal Processes", Journal of Computer Science \& Technology JCS\&T, vol. 5, no. 1, pp. 37 - 43, 2005.

[12] X. Jinqiang and F. Ziping, "A Novel Selfadaptive Fuzzy-PID Controller for Temperature Control in Variable Refrigerant Volume (VRV) Air Conditioning Systems", Advances in Intelligent Systems Research ISKE, 2007. 


\section{con-ciencias}

[13] M. Frank y R. Herbit, "Model based desing of a fuzzy temperature control for a steam generator", EUROSIMS-95, pp. 1137 1142, 1995.

[14] C. Peña, Coevolucionary Fuzzy Modeling. Springer-Verlag Berlin Heidelberg, 2004.

[15] R. Dubisch, Lattices to Logic. Blaisdell Publishing Company, 1964.
[16] A. Gersnoviez, I. Baturone y F. Moreno, "Extracción de Bases de Reglas Simples y Lingüísticamente Interpretables", XIII Congreso Español de Tecnologías y Lógica Fuzzy: ESTYLF, pp. 111-116, 2006.

[17] B. Davey, H. Priestley, Introduction to Lattices and Order. Cambridge University Press, 2002. 